\title{
Using wide biological pores to cap and contain the COVID-19 spike protein
}

\author{
G. Sampath
}

\begin{abstract}
Geometric analysis shows that the spike (S) protein in the COVID-19 virus (SARS-Cov-2) can fully or partially enter into the channel of a wide biological pore like perforin (PFN) or streptolysin (SLO) when the latter is anchored in a bilayer lipid membrane. The PFN channel is a $\beta$ barrel formed from multiple monomers, for example a $\sim 14 \mathrm{~nm}$ diameter channel is formed from 22 monomers. Coincidentally the wide canopy of S (which has three identical chains) has an enclosing diameter of $\sim 14 \mathrm{~nm}$. While inside the channel peripheral residues in the canopy may bind with residues on the pore side of the barrel. If there are no adverse cross-reactions this would effectively prevent S from interacting with a target cell. Calculations with data obtained from PDB and other sources show that there are $\sim 12$ peripheral residue triples in $\mathrm{S}$ within a circle of diameter $\sim 14 \mathrm{~nm}$ that can potentially bind with 22 exposed residues in each barrel monomer. The revised Miyazawa-Jernighan matrix is used to calculate the binding energy of canopy-PFN barrel residue pairs. The results show a large number of binding pairs over distances of up to $38 \AA$ into the pore. This geometric view of capture and containment points to the possibility of using biological pores to neutralize SARS-Cov-2 in its many variant forms. Some necessary conditions that must be satisfied for such neutralization to occur are noted. A wide pore (such as PFN or SLO) can also be used in an electrolytic cell to detect the presence of SARS-Cov-2, which would cause a large-sized blockade of the base current (the ionic current in a fully open pore). It can further be used to quantify the virus level in the sample. Solid-state pores, which have several advantages over biological ones, can be used instead; immune rejection is not an issue and there is no need for the spike or the virus to bind to the pore.
\end{abstract}

\section{Introduction}

The first step in the infection of a host cell by the COVID-19 virus is usually the attachment of the spike (S) protein jutting out of the virus to the ACE2 receptor protein on the membrane of the target cell [1,2]. Many antiviral drugs are designed to neutralize $\mathrm{S}$ by preventing such attachment. Broadly there are three approaches to counter the virus [3]: 1) neutralize the receptor-binding domain (RBD) in S by targeting residues in it [4];2) block pockets in other domains of S [5]; 3) directly deactivate the ACE2 receptor [6]. The inhibiting agent may be one of the following depending on the approach used: antibody proteins [7], peptides [8,9], nanobodies [10], small molecules [11]. Here the possibility of capping and containing $\mathrm{S}$ inside a biological pore [12] is explored by looking at the geometries of S and the pore and considering how $\mathrm{S}$ can enter the pore and be retained inside the pore channel. Such an approach can in principle deactivate the RBD as well as any other pockets that may be present in the other domains of S. As it is based primarily on physical containment it is likely to be indifferent to local mutations or homological differences among variants, evolutionary or otherwise,. The sequences of S and the pore barrel are examined for contact residues in $\mathrm{S}$ and binding sites on the inner wall of the channel. Data are presented for the SARS-Cov2 virus. Additionally a wide pore in electrolytic cell (e-cell) can be used to detect the virus in a sample and also quantify its level in the sample. Solid-state pores also can be used for detection and quantification, in this respect they have some notable advantages over biological ones.

\section{Geometry of the COVID-19 spike protein}

The SARS-Cov-2 (COVID-19) virus is a spherical particle with a protein membrane whose interior contains a protein capsid enclosing a single RNA string that codes for the virus. The membrane surface has about 25-40 spikelike protrusions, each of which is a protein with three identical sequence chains $\mathrm{A}, \mathrm{B}$, and $\mathrm{C}$ forming a mushroom shape with a canopy and a stem. There are two domains in the spike: S1, the canopy consisting of a receptor binding domain (RBD) and a non-terminal domain (NTD); and S2, below the canopy, which directs fusion of the virus to a target cell prior to invading it. The RBD may be closed or open outward to provide a binding site to the ACE2 receptor that is located on the surface of a target cell [1].

Here the focus is on the geometry of the SARS-Cov-2 spike protein. Figure 1 shows plan and side views of 6VXX. The two graphics were obtained from RasMOL views and are annotated to include other pertinent information, see below.

The geometrical properties of the spike can be studied in terms of its extent, which can be calculated in a number of ways, including such well-known methods as minimum enclosing ellipsoid, minimum enclosing right cylinder, and minimum enclosing box or parallelepiped, all of which are global in character [13]. Here attention is focused on the diameter of the canopy so that it can be related to biological pores, which are roughly symmetric cylinders. To this end structure data for the spike protein (PDB id: 6VXX) was downloaded from the RCSB website and atomic coordinates extracted; the results can be found in Supplementary File 1. The centroid of the three C $\alpha$ atoms of each 
residue triple in the three chains was calculated. From it the smallest enclosing diameter circle and the distances of the centroids from the centroids of the topmost and bottommost residues were computed. The results for the 19 largest diameter triples are shown in Table 1. Of these the eight residue triples with diameter within $\sim 15 \AA$ of $14 \mathrm{~nm}$ are shaded.
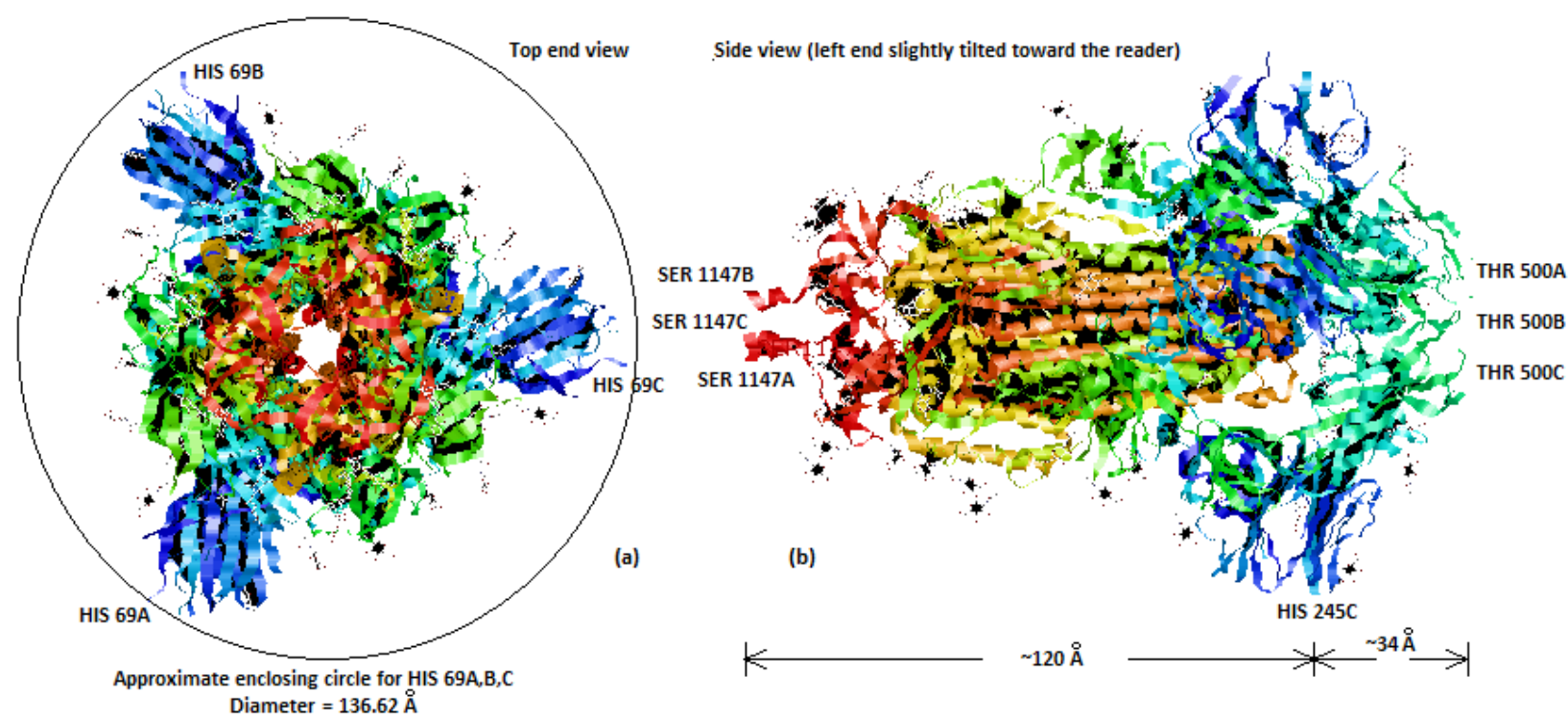

Figure 1. (a) Plan view of spike protein structure looking down from canopy side; smallest enclosing circle for all residues in spike protein is shown. (b) Side view of spike protein; distances of widest diameter residue (HIS 245 A, B, C) to tip of spike (residue SER 1147 A, B, C) and to top of canopy (residue 500 THR A, B, C) are shown.

Table 1. 19 triples (residues in A, B, C) with the largest enclosing diameters; the eight largest are shaded.

\begin{tabular}{|c|c|c|c|}
\hline Residue & $\begin{array}{c}\text { Residue number in } \\
\text { sequence ( } \mathrm{A}, \mathrm{B} \text {, and } \mathrm{C} \\
\text { chains) }\end{array}$ & $\begin{array}{l}\text { Diameter of covering } \\
\text { circle for triple }(\AA)\end{array}$ & $\begin{array}{l}\text { Distance from spike tip } \begin{array}{l}\text { Distance from top of } \\
\text { (residue triple sequence } \\
\text { canopy (residue triple }\end{array} \\
\text { number } 1147, \text { SER) }(\AA) \text { sequence number } 500\end{array}$ \\
\hline
\end{tabular}

$\begin{array}{ccccc} & & & & \text { THR })(\AA) \\ \text { HIS } & 69 & 136.62 & 113.56 & -42.24 \\ \text { HIS } & 245 & 135.35 & 120.5 & -35.29 \\ \text { VAL } & 143 & 130.35 & 123.07 & -32.72 \\ \text { ILE } & 68 & 130.18 & 112 & -43.79 \\ \text { LEU } & 244 & 128.15 & 120.6 & -35.19 \\ \text { GLY } & 142 & 126.62 & 124.93 & -30.87 \\ \text { SER } & 98 & 125.87 & 112.09 & -43.7 \\ \text { LYS } & 97 & 124.24 & 108.43 & -47.37 \\ \text { ALA } & 67 & 122.91 & 113.01 & -42.78 \\ \text { VAL } & 213 & 122.21 & 101.65 & -54.15 \\ \text { ALA } & 243 & 121.72 & 120.12 & -35.67 \\ \text { PHE } & 186 & 121.64 & 104.04 & -51.75 \\ \text { ARG } & 214 & 120.38 & 100.68 & -55.12 \\ \text { LYS } & 187 & 120.09 & 103.83 & -51.96 \\ \text { ILE } & 100 & 119.79 & 115.58 & -40.21 \\ \text { ALA } & 263 & 119.45 & 110.66 & -45.13 \\ \text { ASN } & 211 & 119.38 & 98.6 & -57.19 \\ \text { ALA } & 123 & 119.26 & 120.33 & -35.47 \\ \text { ASN } & 99 & 119.19 & 113.96 & -41.84\end{array}$

Figure 2 shows the projections of the 19 triples on to the XY plane. Note the clustering of the projection points, the result of 3-way symmetry in the structure of the protein (see Figure 1a). 


\section{Geometry of some biological pores}

Pores play a major role in biological function, which may be benign or malign. Proteinaceous pores in the membrane of biological cells allow transport in one direction or the other of particles of various sizes, from $\mathrm{Ca}^{+}$ions to large proteins. Such transport is usually benign and beneficial to normal cellular functions. Pores may also be formed by viruses to attack healthy cells. There are many pore-forming proteins in nature that are toxins and function by puncturing the cell membrane to cause cell death [14 ]. On the other hand such an intrinsically malign property has been turned to advantage in vitro (and in silico) by using these pores as analytical tools in a wide range of applications, including sequencing/identification of biomolecules (DNA, protein, oligomers, peptides) [15,16], analysis of species [17], protein conformation and folding [18 ], and protein dynamics [19].

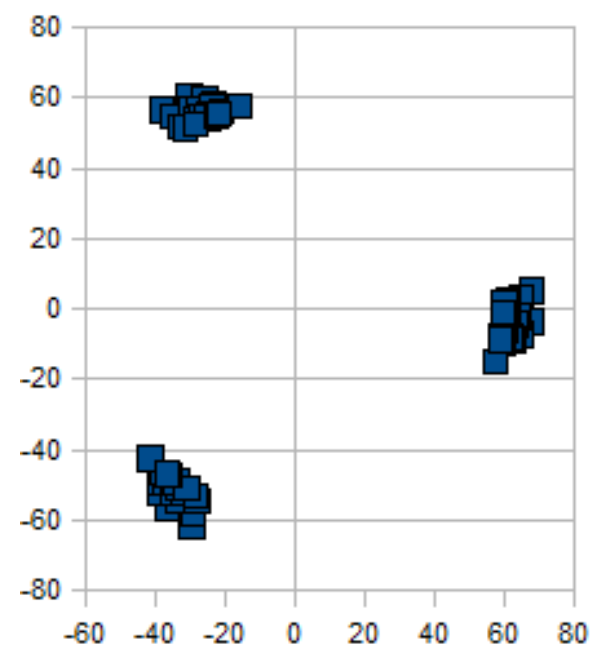

Figure 2. Projections onto XY plane of the 19 largest diameter residue triples in canopy of S

When the naturally occurring protein alpha hemolysin is added to an electrolytic cell (e-cell) in which a lipid bilayer separates two chambers cis and trans, it causes a pore to form through the membrane [20]. If an analyte like DNA or protein is added to the cis chamber and a potential difference is applied across cis and trans the analyte translocates through the pore to trans thereby causing changes in the pore current. These changes can be measured and used to identify bases in the DNA sequence, residues in the protein sequence, or the DNA or protein as a whole. Such pores usually have diameters of $\sim 1-3 \mathrm{~nm}$. Biological pores [21,22], however, have a wide range of diameters, going from 1 $\mathrm{nm}$ all the way up to the micron range. In the present context wide pores with diameters in the $10-250 \mathrm{~nm}$ range are of immediate interest .

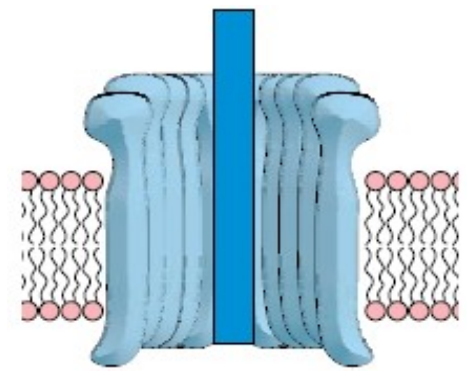

Figure 3. Cutaway view of PFN pore in a bilayer lipid membrane. (Extracted from Figure 1 in [26].)

Over the last few years, wide diameter pores have studied in depth [23]. Two wide pores of considerable interest are perforin (PFN) and streptolysin (SLO). PFN is a toxin that has been studied in detail in [24-26] and is a multifold pore. That is, it is formed from multiple monomers; for example 22 monomers can form a $14 \mathrm{~nm}$ diameter pore. Likewise SLO is a toxic protein that can form pores with inner diameter of up to $25 \mathrm{~nm}$ [27]. Both PFN and SLO 
can form pores that are full rings or arcs in a giant $\beta$ barrel (a cylindrical structure made up of linked $\beta$ sheets). The relationship between the pore diameter and the number of monomers has been studied in [28].

The present report focuses on the potential use of PFN as a device to cap and contain the spike protein in SARS-

Cov-2. Figure 3 shows a cutaway view of a PFN pore anchored in a bilayer lipid membrane (graphic extracted from Figure 1 in [26]). The structure of the monomer is shown in Figure 4a below. The sequence of residues in the $\beta$ sheet at the bottom of the structure that face the inside of the pore is shown in Figure 4b, the figure is an adaptation from [26].

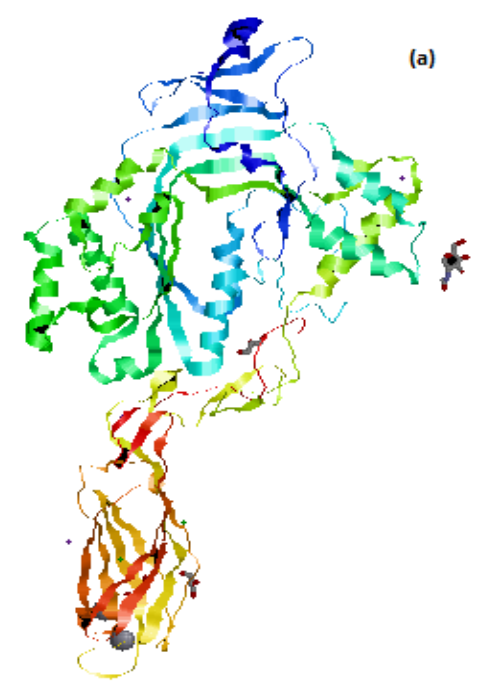

(a)

(b)

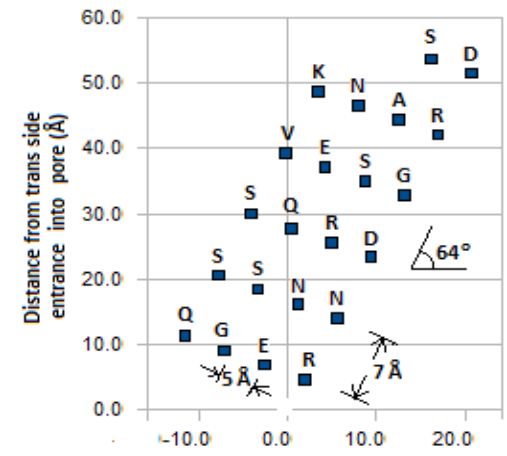

Figure 4. (a) Structure of single monomer of perforin (PFN). (b) Residues facing inside of pore channel

Table 2 shows the 22 residues in the $\beta$ sheet at the bottom of the PFN monomer sorted by $\mathrm{z}$ value. This ordering on $\mathrm{z}$ is used to define a barrel array for use in calculating the total energy of the spike-barrel ensemble.

Table 2. Shows $x$ and $z$ coordinates of residues in Figure 4. Last column shows integer value of $z$.

$\begin{array}{cc}\text { Sorted on } \mathbf{z} & \mathbf{x} \\ \mathrm{R} & 1.9 \\ \mathrm{E} & -2.6 \\ \mathrm{G} & -7.1 \\ \mathrm{Q} & -11.6 \\ \mathrm{~N} & 5.7 \\ \mathrm{~N} & 1.2 \\ \mathrm{~S} & -3.3 \\ \mathrm{~S} & -7.8 \\ \mathrm{D} & 9.4 \\ \mathrm{R} & 4.9 \\ \mathrm{Q} & 0.5 \\ \mathrm{~S} & -4.0 \\ \mathrm{G} & 13.2 \\ \mathrm{~S} & 8.7 \\ \mathrm{E} & 4.2 \\ \mathrm{~V} & -0.3 \\ \mathrm{R} & 17.0 \\ \mathrm{~A} & 12.5 \\ \mathrm{~N} & 8.0 \\ \mathrm{~K} & 3.5 \\ \mathrm{D} & 20.8 \\ \mathrm{~S} & 16.3 \\ & \end{array}$

$\mathbf{z}$
4.7
6.9
9.1
11.3
14.0
16.2
18.4
20.6
23.4
25.6
27.8
30.0
32.8
35.0
37.1
39.3
42.1
44.3
46.5
48.7
51.5
53.7

Truncated $z$

9.1

14.0

16.2

18.4

23.4

25.6

27.8

32.8

35.0

37.1

44.3

46.5

51.5

53.7

Figure 5 shows the PFN $\beta$ barrel schematically as a cut and unrolled cylinder laid out flat. 


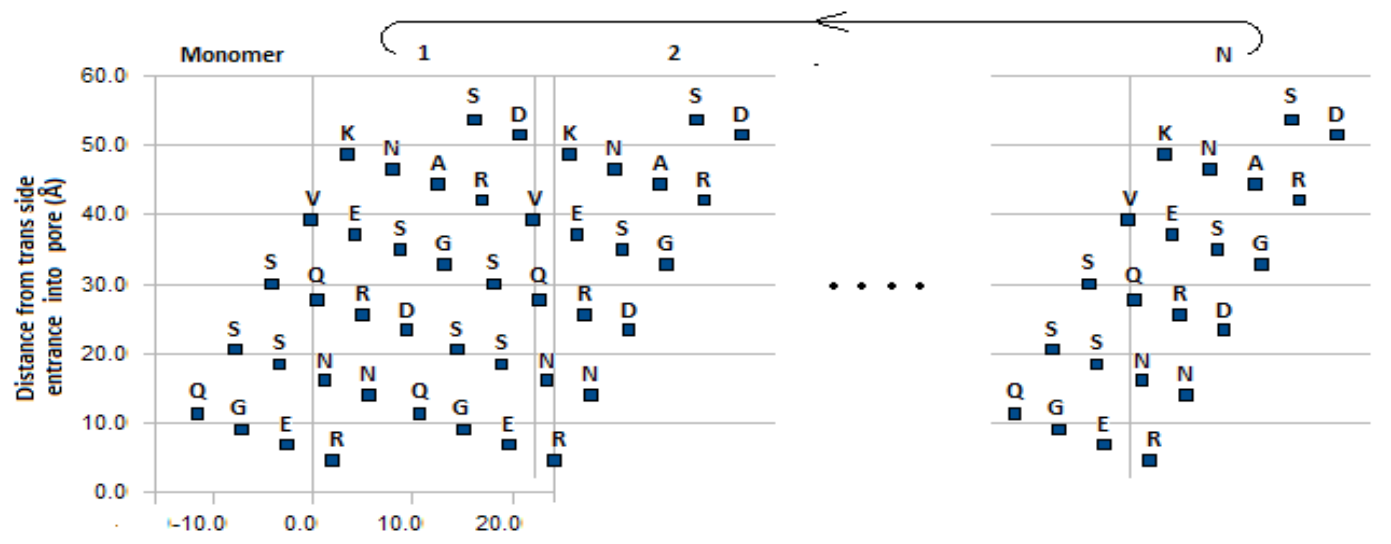

Figure 5. Schematic of $\beta$ barrel in PFN pore shown with the barrel cut, unrolled, and laid flat. Residues with negative $\mathrm{x}$ values are rolled over to the right end (not shown).

\section{Capping and containing the spike protein with perforin}

Given the compatible diameters of the canopy of the spike protein and the 22-monomer PFN pore, it may be possible to use the pore to cap and contain the spike protein. Figure 6 shows how this could happen. The graphic is obtained by overlaying the spike protein graphic in Figure $1 \mathrm{~b}$ on to Figure 3. Notice that the spike can be positioned inside the pore at any horizontal angle in the XY plane. Thus as the spike travels through the pore its canopy residues come up against different pore residues depending on the spike rotation angle. The total energy of the spikebarrel ensemble will depend on the contact energy between spike and pore residues.

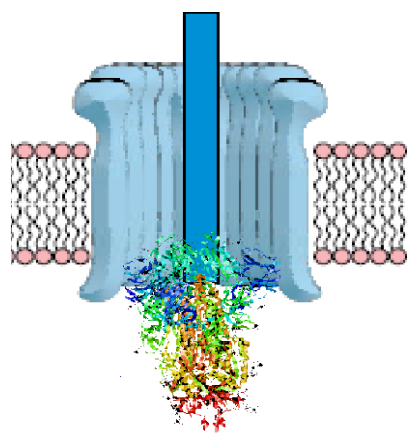

Figure 6. Conceptual view of spike protein as it enters PFN pore from trans side of pore. View obtained by superposing spike protein in Figure 1b over cutaway view of pore in Figure 3.

Protein folding and protein-protein interaction (PPI) models are often based on considering the affinity between pairs of residues, in the same protein in the case of folding, or different proteins in the case of PPI. Many bindingenergy-based models use the Miyazawa-Jernighan (MJ) matrix introduced in [29] and revised in [30] (see Supplementary File 2). Other more recent studies include [31-33]. While the MJ matrix has been most widely used in protein folding models, it has also been used in PPI models, for example see [34].

\section{Mapping the barrel and the canopy into arrays}

In the present study the MJ matrix is used to calculate the energy between residues in S and in the PFN barrel. To this end residues in the barrel and canopy are mapped to binary arrays of dimension $\mathrm{L} \times \mathrm{W}$ and $\mathrm{H} \times \mathrm{W}$ respectively, in which a row or column corresponds to a distance of $1 \AA$. Here $\mathrm{L}$ is the $\mathrm{z}$ value of the highest residue in Figure 5 and $\mathrm{W}=\pi \mathrm{d}$ is the circumference of the pore with $\mathrm{d}=$ diameter of the pore. If there is a residue at $(\mathrm{z}, \mathrm{x})$ in Figure 5 (negative $\mathrm{x}$ values are made positive by adding $\mathrm{W}$ to them) then the entry at row trunc(z) and column trunc(x) in the barrel matrix is 1 and 0 otherwise. Similarly if there is a residue in the canopy at height $\mathrm{z}$ (see Table 1) with angle $\theta$ (degrees) in the XY plane (see Figure 3 ) then the entry in the canopy matrix at row trunc(z) and column $\mathrm{x}=$ $\operatorname{trunc}(\theta \mathrm{W} / 360)$ is 1 . The contents of the two binary arrays are shown in Figure 7 with $\square$ or $\mathbf{m}$ used in place of 1. 

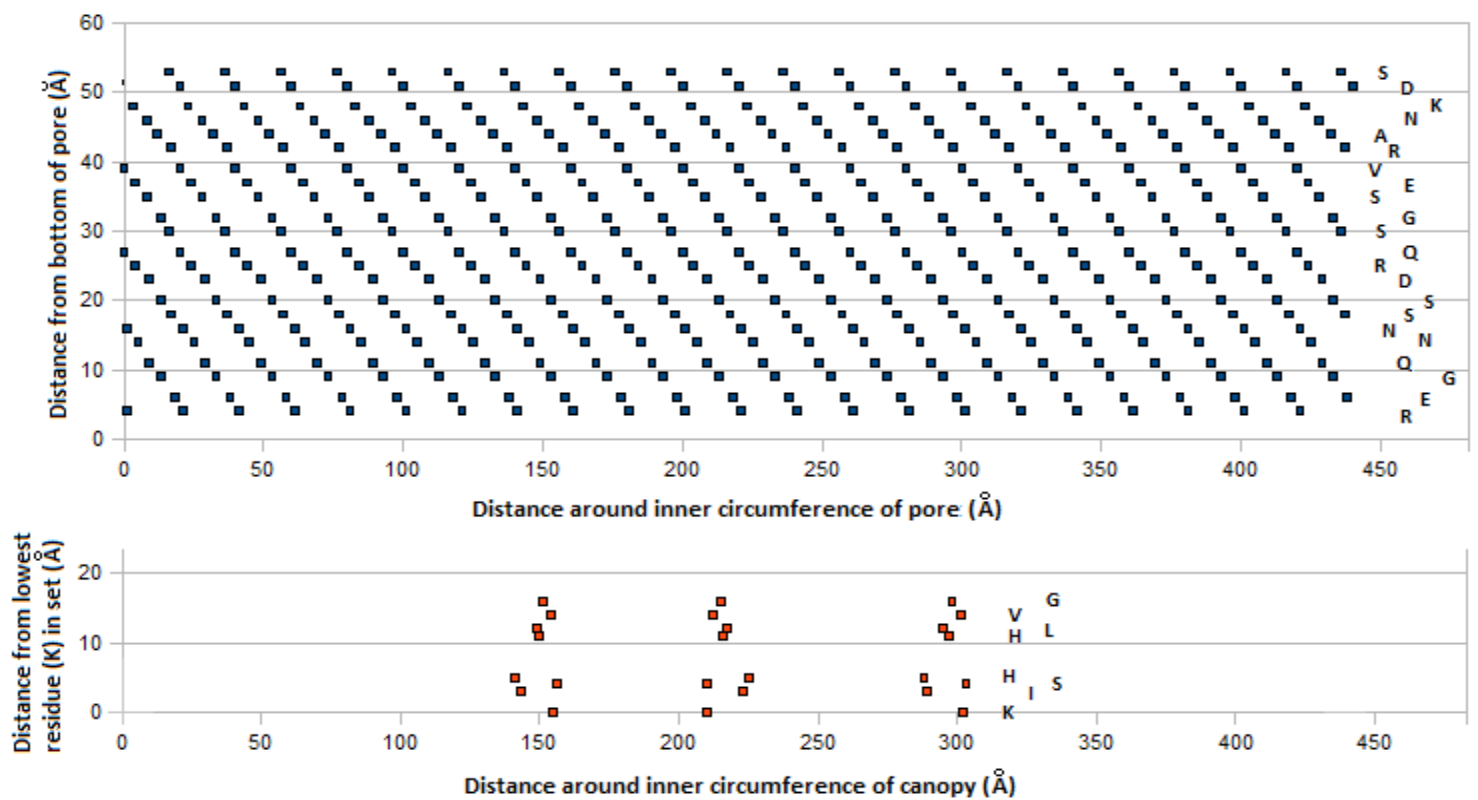

Figure 7. Contents of binary barrel and canopy matrices. $\square=\mathbf{m}=1$; 0s not shown. Letters on the right are 1-letter names of residues on corresponding row.

\section{Binding energy of pore-canopy ensemble}

1) A simple energy model considers a canopy residue to be bound to (or coincident with) a pore residue if the corresponding entries in the barrel and canopy arrays are both 1 . To determine binding pairs, the two arrays are AND-ed. Entries in the AND-ed result that are 1 corresponding to binding pairs $\left(\mathrm{R}_{\mathrm{B}}, \mathrm{R}_{\mathrm{S}}\right)$, where $\mathrm{B}$ and $\mathrm{S}$ refer to the barrel and the spike. This is repeated for every circular position of the canopy by doing a circular rotation of the canopy array 1 column to the right; there are thus $\mathrm{W}$ instances of the canopy array. The canopy array is then moved 1 row up on the barrel array and the procedure repeated $\mathrm{L}-\mathrm{H}+1$ times.

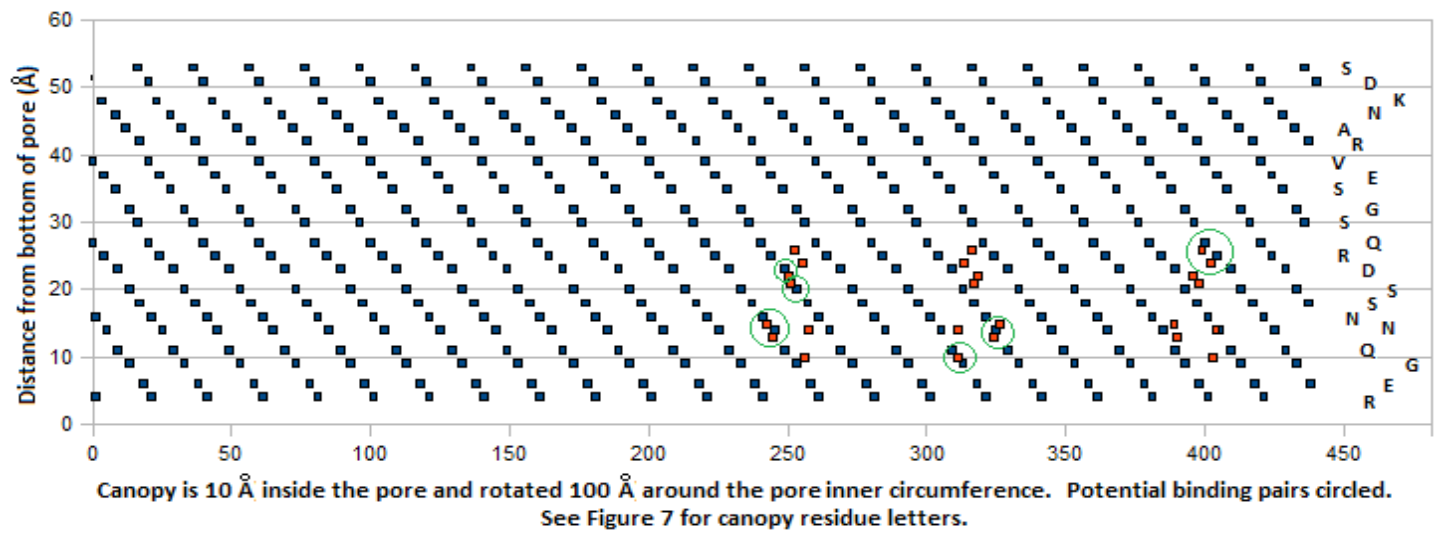

Figure 8. Canopy array ( $\square)$ superimposed on barrel array ( $\square)$. Spike has moved $10 \AA$ into pore and rotated 80 degrees (with respect to Figure 3).

Figure 8 shows an example of the spike-barrel ensemble in which the spike has traveled $z_{\text {in }}=10 \AA$ into the pore and rotated $100 \AA$ around the inner circumference of the pore $(\approx 80$ degrees with reference to the angular position of the spike in Figure 3). Corresponding to this movement the canopy array has moved up 10 rows and shifted right by 100 columns relative to the barrel array.

2) A more involved model considers binding to occur between a canopy residue at $\left(\mathrm{z}+\mathrm{Z}_{\mathrm{in}}, \mathrm{X}\right)$, where $\mathrm{z}_{\mathrm{in}}=$ distance of 
spike up the pore, and a barrel residue at any of the following locations in the barrel array: $\left(z+z_{\text {in }}, x\right),\left(z+z_{\text {in }}+1, x\right)$, $\left(\mathrm{z}+\mathrm{z}_{\mathrm{in}}-1, \mathrm{x}\right),\left(\mathrm{z}+\mathrm{z}_{\mathrm{in}}, \mathrm{x}+1\right),\left(\mathrm{z}+\mathrm{z}_{\mathrm{in}} \cdot \mathrm{x}-1\right)$. The total energy is then the sum of the energies in all applicable cases.

Figure 9 shows the results of the two models.
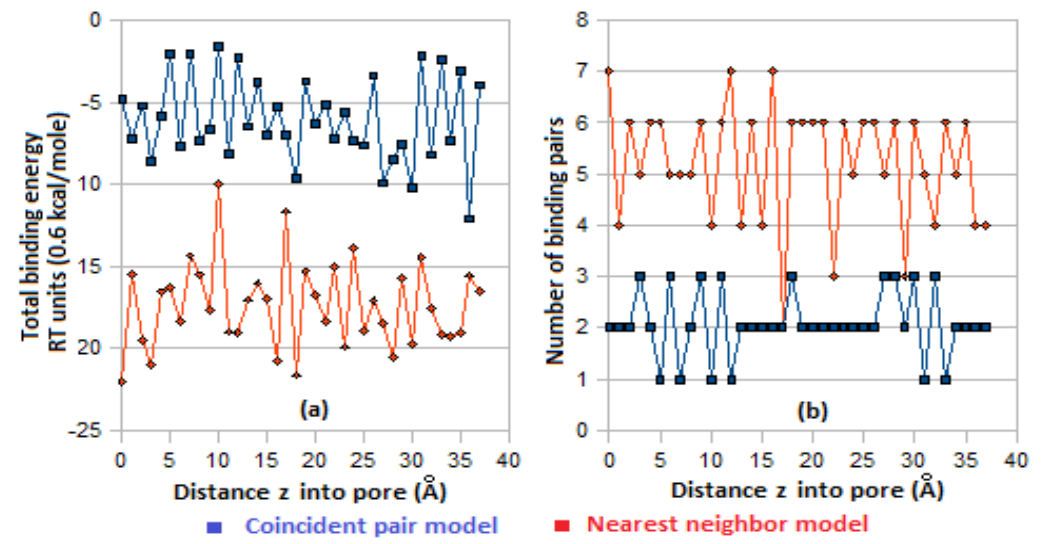

Figure 9. (a) Binding energy of pore-canopy ensemble as a function of the distance $z_{\text {in }}$ traveled into the pore by the spike; (b) Number of binding pairs vs distance $z_{\text {in }}$.

\section{SARS-Cov-2 virus detection and quantification with wide pores in an electrolytic cell}

Wide pores may also be useful in detecting the presence of the Covid-19 virus in a sample. Currently used detection procedures are based on RT-PCR applied to viral RNA or on testing for the presence of antibodies in a serum sample. The former applies to current infections, the latter to prior ones [35]. Other approaches are based on biosensors that use electrical or optical methods [36]. Here the capping of a spike protein by a wide biological pore as described above suggests an electrical method for the detection of the SARS-Cov-2 virus. The e-cell mentioned earlier can be used to measure the current that flows through a pore like PFN or SLO when an electrical potential is applied across the cis and trans chambers. The pore used for detection does not have to be biological as it is in the case of neutralization of the virus. There is no need to consider immune response, so solid-state pores, which have several advantages over biological ones, can be used instead. They can be fabricated with diameters ranging from sub-nanometer to as high as $200 \mathrm{~nm}$ [37]. In the following the use of solid-state pores is assumed.

If a buffered sample is introduced into cis or trans, the virus (whose structural proteins carry an electrical charge) is drawn toward the pore and blocks it nearly fully. As a result the base current, that is, the current through the pore when there is no analyte in it, drops to near zero. This binary change in the pore current signals the presence of a large blocking molecule. If the sample does not contain any components of comparable or larger size then this state change can be used to detect the presence of the virion in the sample. These larger particles can be filtered out by using micro/ultra filtration methods [38] prior to entry of the virus and other smaller particles (such as DNA, RNA, etc.) into the e-cell. Table 3 lists the electrical charge carried by the four (unfolded) structural proteins M, N, E, and $\mathrm{S}$ in the virus at different $\mathrm{pH}$ values of the solution (electrolyte); the effective charge on the folded protein is less because of unexposed residues. By adjusting the $\mathrm{pH}$ of the solution this effective charge on all the structural proteins can be made to have the same polarity. This ensures electrophoretic translocation of the virus particle through the pore with a voltage across the pore of appropriate polarity (see Figure 10 below).

Table 3. Electrical charge carried by the four structural proteins (unfolded) of SARS-Cov-2 for different $\mathrm{pH}$ values

\begin{tabular}{|c|c|c|c|c|}
\hline \multirow{2}{*}{$\begin{array}{c}\mathrm{pH} \\
\text { value }\end{array}$} & \multicolumn{4}{|c|}{$\begin{array}{l}\text { Electrical charge carried by (unfolded) structural } \\
\text { protein in SARS-Cov- } 2\left(\times 1.6 \times 10^{-19} \text { coulomb) }\right.\end{array}$} \\
\hline & M & $\mathrm{N}$ & $\mathrm{E}$ & $\mathrm{S}$ (monomer) ${ }^{(1)}$ \\
\hline 3.0 & 12.19 & 12.61 & 2.37 & 8.79 \\
\hline 5.0 & 8.14 & 5.43 & 1.86 & -7.79 \\
\hline 7.0 & 8.14 & 5.43 & 1.86 & -7.79 \\
\hline 9.0 & 4.48 & 1.72 & -1.01 & -13.88 \\
\hline 12.0 & -14.43 & -18.66 & -8.63 & -45.28 \\
\hline
\end{tabular}


Figure 10 shows the two stages: micro/ultra filtration under pressure and current blockade in an e-cell with an electrical potential across the pore membrane. A buffered sample is subjected to hydraulic pressure in an ultrafilter, which allows particles of size approximately $\leq$ the virus diameter to filter through (Figure 10a). The filtrate containing virus particles is injected into the cis chamber of an e-cell with a membrane containing a pore that allows particles of size $<$ the virus diameter to translocate into the trans chamber through a combination of diffusion and electrophoresis (Figure 10b). This results in the virus particle (if present ) blocking the pore resulting in near total blockade of the pore current (Figure 10d). This is a binary measurement: if the e-cell current drops to near zero, the presence of the virus is indicated. However the possibility of a false positive exists as there could be other particles whose diameter is about the same as the virus diameter. On the other hand if there is no drop in the pore current over a time of a few msecs and there is no adsorptive loss in the filtering stage or at the surface of the membrane on the cis side, absence of the virus in the sample is indicated. In this case, the probability of a true negative is $100 \%$.

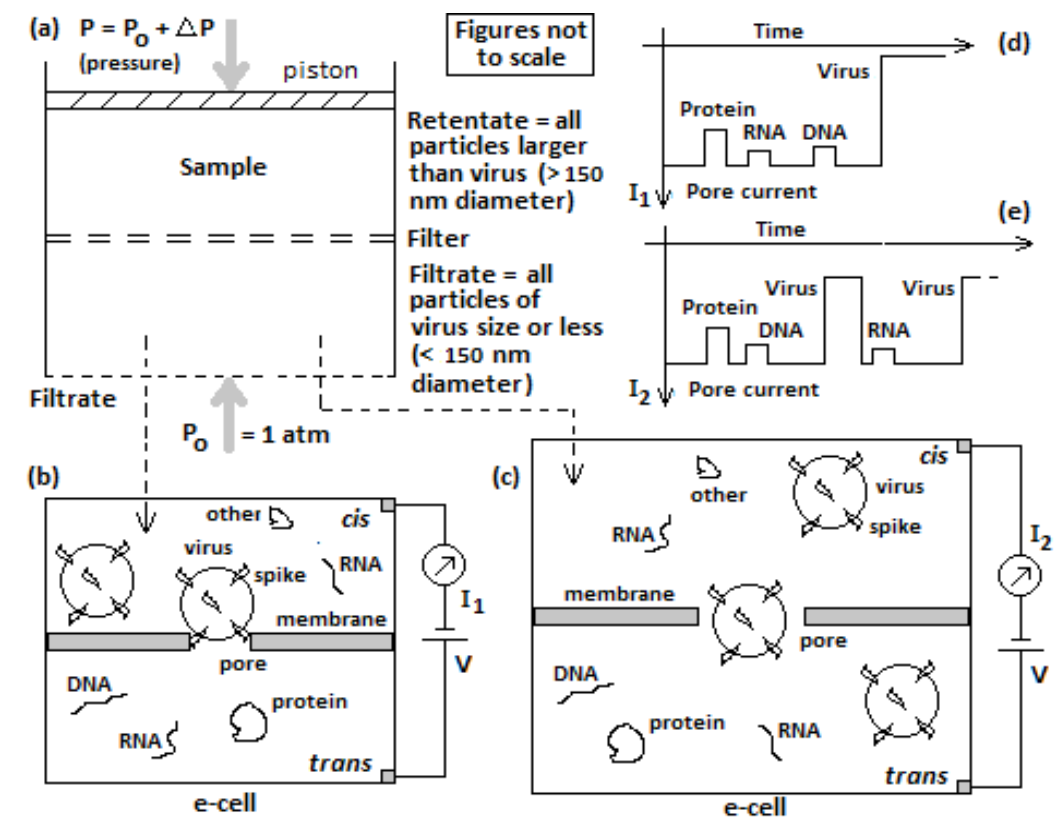

Figure 10. Detection and quantification of Covid-19 virus in sample. (a) Buffered sample filtered through ultrafilter to allow particles of size approximately $\leq$ virus diameter to filter through; (b) Filtrate containing virus particles enters cis chamber of electrolytic cell (e-cell) with pore that allows particles of size $<$ virus diameter to translocate into trans chamber via diffusion and electrophoresis; virus particle blocks pore almost completely when attempting to enter pore, causes near total blockade, see (d); (c) Filtrate enters e-cell with pore of diameter > virus diameter, virus translocates through pore to cause substantial and measurable blockade; (d) Representational graph showing blockades caused by particles of various size in (b), with near total blockade of pore by virus that more or less shuts off pore current; (e) Representational graph showing blockades caused by particles of various sizes in (c), each translocating virus particle causes blockade of size considerably different from those due to other particles (like DNA, RNA, etc.); blockade count can be used to quantify virus in sample.

The above development has been limited to detecting the virus. To both detect and quantify the virus, an e-cell with pore diameter greater than the virus diameter can be used to allow all virus particles (along with other particles in the sample) to translocate through the pore (Figure 10c). The virus particles, because of their large diameter (relative to the pore diameter), cause blockades whose size is substantial, easily measurable, and considerably different from blockades due to other smaller particles like DNA, RNA, protein, etc. Each translocating virus causes a blockade; by counting these multiple blockades (Figure 10e) the virus level in the sample can be quantified.

\section{Filtration time}

Consider an ultrafilter membrane with an array of $40 \times 40$ pores of diameter $\mathrm{d}=150 \mathrm{~nm}$ and length $\mathrm{L}=10 \mathrm{~nm}$, pressure $\Delta \mathrm{P}=2.0 \mathrm{~atm}$, and buffer (dilute salt solution) viscosity $\eta \approx 0.001 \mathrm{~Pa}$.sec. The Poiseuille flow rate is $\mathrm{Q}=$ 
$\pi(\mathrm{d} / 2)^{4} \Delta \mathrm{P} / 8 \eta \mathrm{L} \approx 10^{-9} \mathrm{~m}^{3} / \mathrm{s}$. A buffered sample volume of $1 \mathrm{cc}$ can be filtered in about 7 minutes.

Translocation time of virus in e-cell

When the filtrate is added to the cis chamber of the e-cell the blockade detection due to a single virus particle will occur in a few $\mu$ secs. This requires a detection bandwidth of a few $\mathrm{MHz}$, which may lead to problems because of high frequency noise. The bandwidth can be reduced to a few Khz by using a bi-level positive-negative voltage profile across the e-cell pore and adjusting the $\mathrm{pH}$ of the solution. To ensure delivery of the analyte in the cis chamber to the pore, hydraulic pressure can be used. See [39] for more information.

The combined filtration and translocation time is a few minutes, so the detection (and quantification) process is fast. As noted earlier, with this method the probability of a true negative is $100 \%$ if there is no adsorption in the filter membrane or at the surface of the pore membrane in the e-cell. The true positive probability depends on the 'impurity' content, where an 'impure particle' is any particle of approximately the same size as the SARS-Cov-2 virus. The method is based on currently available filtration technology and uses only electrical measurement. It does not involve any complex time-consuming procedures or expensive equipment and is fairly easy to translate into a hand-held device for use in point-of-care (POC) diagnosis.

\section{Discussion}

Translation of the cap-and-contain method proposed above into an anti-viral protocol needs to consider a wide range of issues. Some of them are: a) developing an appropriate method of delivery; b) determining the right dosage; and c) ensuring that the integrity of the bilayer lipid membrane is not diminished after delivery. The last is especially important because most biological pores, including PFN and SLO, are toxins in their natural form [14]; the cure should not be worse than the contagion. Some practical and other relevant issues are noted next.

1) Improved binding between $S$ and the pore channel can be achieved by artificially introducing residues into PFN through selective mutagenesis at a desired set of positions [40].

2) Notice that with the entire canopy of the spike capped by the pore, other pockets in the spike that can potentially bind with the target cell are effectively closed off.

3 ) The maximum number of coincidences between a residue on a barrel row and a residue on a canopy row (see Figure 8) is determined by the relationship between the number of monomers (22) and the number of canopy residues on any row (3). This number is minimal because 22 and 3 are relatively prime. It can be increased by using 21 or 24 monomers, but this also affects the diameter of the pore and hence its relationship to the diameter of a canopy residue triple.

4) Since capping is largely a physical process, whether the RBD (or any other domain) is conserved or not may not be an issue.

5) One of the problems with attempts to neutralize the spike is that the latter is covered with a glycan shield that thwarts attempts by antibodies to detect it [41]. Such evasion by the virus may not be an issue when it is capped by a wide pore as long as there are no other cross-reactions.

6) An interesting property of the SARS-Cov-2 virus is that the spherical membrane appears to be quite robust and resists mechanical attempts to change its shape [42]. Thus it is unlikely to squeeze through the pore of an e-cell.

7) When the pore (anchored in the bilayer lipid membrane) enters the extracellular environment, like any other foreign body it can be expected to trigger an immune response, typically in the form of antibodies. As long as these antibodies do not block the pore or otherwise adversely impact the pore the latter's ability to cap and contain the spike will likely remain unaffected.

8) Other electrochemical methods for detection of the SARS-Cov-2 have been reported recently; see [43] for example.

9) A cap-and-contain approach somewhat like the one described above has been used earlier in a study of Huntingdon disease in which aggregation of a mutant protein (mHTT) is inhibited by a chaperonin complex (TriC), which caps the tips of HTT fibrils and encapsulates mHTT oligomers [44].

\section{References}

[1] David Goodsell, "Molecule of the Month: SARS-CoV-2 Spike”, June 2020, RCSB.org. doi: 10.2210/rcsb_pdb/ mom_2020_6

[2] J. Lan, J. Ge, J. Yu, S. Shan, H. Zhou, S. Fan, Q. Zhang, X. Shi, Q. Wang, L. Zhang, and X. Wang, "Structure of the SARS-CoV-2 spike receptor-binding domain bound to the ACE2 receptor", Nature, 581, 215-229, 2020. doi:

$10.1038 / \mathrm{s} 41586-020-2180-5$ 
[3] S. Pomplun, "Targeting the SARS-CoV-2-spike protein: from antibodies to miniproteins and peptides", RSC Med. Chem., 12, 197-202, 2021.

[4] G. Zhang, S. Pomplun, A. R. Loftis, X. Tan, A. Loas, and B. L. Pentelute, "Investigation of ACE2 N-terminal fragments binding to SARS-CoV-2 Spike RBD", bioRxiv preprint, June 17, 2020. doi: 10.1101/2020.03.19.999318

[5] S. Yazdani, N. De Maio, Y. Ding, V. Shahani, N. Goldman, and M. Schapira, "Genetic variability of the SARS-

CoV-2 pocketome", J. Proteome Res. 2021. doi: 10.1021/acs.jproteome.1c00206

[6] G. Wang, M.-L. Yang, Z.-L. Duan, F.-L. Liu, L. Jin, C.-B. Long, M. Zhang, X.-P. Tang, L. Xu, Y.-C. Li, P. M. Kamau, L. Yang, H.-Q. Liu, J.-W. Xu, J.-K. Chen, Y.-T. Zheng, X.-Z. Peng, and R. Lai,"Dalbavancin binds ACE2 to block its interaction with SARS-CoV-2 spike protein and is effective in inhibiting SARS-CoV-2 infection in animal models", Cell Research, 31, 17-24, 2021. doi: 10.1038/s41422-020-00450-0

[7] C. O. Barnes, C. A. Jette, M. E. Abernathy, K.-M. A. Dam, S. R. Esswein, H. B. Gristick, A. G. Malyutin, N. G. Sharaf, K. E. Huey-Tubman, Y. E. Lee, D. F. Robbiani, M. C. Nussenzweig, A. P. West Jr., and P. J. Bjorkman, "SARS-CoV-2 neutralizing antibody structures inform therapeutic strategies", Nature, 588, 682-701, 2020. doi: 10.1038/s41586-020-2852-1

[8] M. Tonk, D. Ružek, and A. Vilcinskas, "Compelling evidence for the activity of antiviral peptides against SARSCoV-2", Viruses, 13, 912, 2021. doi: 10.3390/v13050912

[9] W. Lin, J. Rafeya, V. Roschewitz, D. Smith, A. Keller, and Y. Zhang, "Peptide scanning of SARS-CoV and SARS-CoV-2 spike protein subunit 1 reveals potential additional receptor binding sites", bioRxiv preprint, August 16, 2021. doi: 10.1101/2021.08.16.456470

[10] T. F. Custódio, H. Das, D. J. Sheward, L. Hanke, S. Pazicky, J. Pieprzyk, M. Sorgenfrei, M. Schroer, A. Gruzinov, C. Jeffries, M. Graewert, D. Svergun, N. Dobrev, K. Remans, M. A. Seeger, G. M. McInerney, B. Murrell, B. M. Hällberg, and C. Löw, "Selection, biophysical and structural analysis of synthetic nanobodies that effectively neutralize SARS-CoV-2", bioRxiv preprint, June 23, 2020. doi: 10.1101/2020.06.23.165415

[11] D. Bojadzic, O. Alcazar, J. Chen, S.-T. Chuang, J. M. C. Capcha, L. A. Shehadeh, and P. Buchwald, "Smallmolecule inhibitors of the coronavirus spike: ACE2 protein-protein interaction as blockers of viral attachment and entry for SARS-CoV-2", ACS Infect. Dis. 2021, 7, 1519-1534. doi: 10.1021/acsinfecdis.1c00070

[12] H. Bayley and L. Jayasinghe, "Functional engineered channels and pores (Review)", Molecular Membrane Biology, 21, 209-220, 2004.

[13] P. Schneider and D. H. Eberly. Geometric Tools for Computer Graphics. Elsevier, 2002.

[14] H. Bayley, "Piercing insights: Pore-forming proteins", Nature 459, 651-652, 2009.

[15] D. Deamer, M. Akeson, and D. Branton, "Three decades of nanopore sequencing". Nat. Biotechnol. 34, 518$524,2016$.

[16] N. Varongchayakul, J. Song, A. Meller, and M. W. Grinstaff, "Single-molecule protein sensing in a nanopore: a tutorial", Chem Soc Rev. 47, 8512-8524, 2018. doi:10.1039/c8cs00106e

[17] N. Liu, Z. Yang, X. Ou, B. Wei, J. Zhang, Y. Jia1, and F. Xia, "Nanopore-based analysis of biochemical species", Microchim. Acta, 183, 2016, 955-963. doi: 10.1007/s00604-015-1560-2

[18] G. Oukhaled, J. Mathe', A.-L. Biance, L. Bacri, J.-M. Betton, D. Lairez, J. Pelta, and L. Auvray, "Unfolding of proteins and long transient conformations detected by single nanopore recording", Phys. Rev. Lett. 98, 158101, 2007.

[19] S. Schmid and C. Dekker, "Nanopores - a versatile tool to study protein dynamics", Report, TU Delft, 2020.

[20] H. Bayley, "Nanopore sequencing: from imagination to reality", Clin Chem. 61, 25-31, 2015. doi:

10.1373/clinchem.2014.223016

[21] K. Huang, "Engineering biological nanopores for proteomics study", Dissertation, University of Groningen, 2019. doi: $10.33612 /$ diss. 102598418

[22] S. Wang, "Development of New Biological Nanopores and Their Application for Biosensing and Disease Detection", Dissertation, U Kentucky, 2016. doi: 10.13023/ETD.2016.428

[23] H. Watanabe, A. Gubbiotti, M. Chinappi, N. Takai, K. Tanaka, K. Tsumoto, and R. Kawano, "Analysis of pore formation and protein translocation using large biological nanopores", Anal. Chem. 2017. doi:

10.1021/acs.analchem.7b01550

[24] R. H. P. Law, N. Lukoyanova, I. Voskoboinik, T. T. Caradoc-Davies, K. Baran, M. A. Dunstone, M. E.

D'Angelo, E. V. Orlova, F. 'li Coulibaly, S. Verschoor, K. A. Browne, A. Ciccone, M. J. Kuiper, P. I. Bird, J. A.

Trapani, H. R. Saibil, and J. C. Whisstock, "The structural basis for membrane binding and pore formation by lymphocyte perforin", Nature, 2010. doi: 10.1038/nature09518

[25] I. Osinska, K. Popko, and U. Demkow, "Perforin: an important player in immune response", Central Eur. J. Immunol. 39, 109-115, 2014. doi: 10.5114/ceji.2014.42135

[26] M. E. Ivanova, N. Lukoyanova, S. Malhotra, M. Topf, J. A. Trapani, I. Voskoboinik, and H. R. Saibil, "The pore 
conformation of lymphocyte perforin", bioRxiv preprint, July 3, 2021. doi: 10.1101/2021.07.03.450947

[27] H. Yasuga, R. Kawano, M. Takinoue, Y. Tsuji, T. Osaki, K. Kamiya, N. Miki, and S. Takeuchi, "Logic gate operation by DNA translocation through biological nanopores", PLoS ONE 11:e0149667, 2016.

doi:10.1371/journal.pone. 0149667

[28] C. F. Reboul, K. Mahmood, J. C. Whisstock, and M. A. Dunstone, "Predicting giant transmembrane ß-barrel architecture". Bioinformatics, 2012. doi:10.1093/bioinformatics/bts152.

[29] S. Miyazawa and R. L. Jernigan, "Estimation of effective interresidue contact energies from protein crystal structures: quasi-chemical approximation", Macromolecules, 18, 534-552, 1985.

[30] S. Miyazawa and R. L. Jernigan, "Residue-residue potentials with a favorable contact pair term and an unfavorable high packing density term, for simulation and threading", J. Mol. Biol. 256, 1996, 623-644.

[31] J. C. Biro, "Amino acid size, charge, hydropathy indices and matrices for protein structure analysis", Theor. Biol. and Medical Modelling, 3, 15, 2006. doi:10.1186/1742-4682-3-15

[32] A. Vangone, A. M. Bonvin, "Contacts-based prediction of binding affinity in protein-protein complexes", eLife. 2015;4:e 07454

[33] T. Siebenmorgen and M. Zacharias, "Computational prediction of protein-protein binding affinities", Comput Mol Sci., 10:e1448, 2020. doi: 10.1002/wcms.1448

[34] M. Heo, S. Maslov, and E. Shakhnovich, "Topology of protein interaction network shapes protein abundances and strengths of their functional and nonspecific interactions", PNAS, 108, 4258-4263, 2011. doi:

10.1073/pnas. 1009392108

[35] B. D. Kevadiya, J. Machhi, J. Herskovitz, M. D. Oleynikov, W. R. Blomberg, N. Bajwa, D. Soni, S. Das, M. Hasan, M. Patel, A. M. Senan, S. Gorantla, J. McMillan, B. Edagwa, R. Eisenberg, C. B. Gurumurthy, St. P. M.

Reid, C. Punyadeera, L. Chang, and H. E. Gendelman, "Diagnostics for SARS-CoV-2 infections", Nat. Materials, 20, 593-605, 2021. doi: 10.1038/s41563-020-00906-z

[36] J. F. Huggett, J. Moran-Gilad, and J. E. Lee, "COVID-19 new diagnostics development: novel detection methods for SARS-CoV-2 infection and considerations for their translation to routine use", Curr. Opin. Pulm. Med. 27, 155-162, 2021. doi: 10.1097/MCP.0000000000000768

[37] Y. He, M. Tsutsui, Y. Zhou, and X.-S. Miao, "Solid-state nanopore systems: from materials to applications", NPG Asia Materials, 2021. doi: 10.1038/s41427-021-00313-z

[38] M. Ulbricht, "Nanoporous polymer filters and membranes, selective filters", Encyclopedia of Polymeric Nanomaterials, 2015, Springer-Verlag, Berlin. doi: 10.1007/978-3-642-36199-9_357-1

[39] G. Sampath, "A counter-intuitive method for slowing down an analyte in a nanopore and its application to polymer sequencing and analysis", biorxiv preprint, 2021. doi 10.1101/2021.01.11.426231

[40] A. Crnkovic, M. Srnko, and G. Anderluh, "Biological nanopores: engineering on demand", Life 11, $27,2021$. doi: 10.3390/life11010027

[41] O. C. Grant, D. Montgomery, K. Ito, R. J. Woods, "Analysis of the SARS-CoV-2 spike protein glycan shield: implications for immune recognition", bioRxiv preprint, May 1, 2020. doi: 10.1101/2020.04.07.030445

[42] B. Kiss, Z. Kis, B. Pa'lyi, and M. S. Z. Kellermayer, "Topography, spike dynamics, and nanomechanics of individual native SARS-CoV-2 virions", Nano Lett. 21, 2675-2680, 2021.

[43] A. Ramanujam, S. Almodovar, and G. G. Botte, "Ultra-fast electrochemical sensor for point-of-care COVID-19 diagnosis using non-invasive saliva sampling", Processes 9, 1236, 2021. doi: 10.3390/pr9071236

[44] M. C. Darrow, O. A. Sergeeva, J. M. Isas, J. G. Galaz-Montoya, J. A. King, R. Langen, M. F. Schmid, and W. Chiu, "Structural mechanisms of mutant Huntingtin aggregation suppression by the synthetic chaperonin-like CCT5 complex explained by cryoelectron tomography", J. Biol. Chem. 290, 17451-17461, 2015.

Email: sampath_2068@yahoo.com 